\title{
NON-INTEGRABILITY OF CERTAIN HAMILTONIAN SYSTEMS. APPLICATIONS OF THE MORALES-RAMIS DIFFERENTIAL GALOIS EXTENSION OF ZIGLIN THEORY
}

\author{
ANDRZEJ J. MACIEJEWSKI \\ Institute of Astronomy, University of Zielona Góra \\ Lubuska 2, 65-265 Zielona Góra, Poland \\ E-mail: maciejka@astro.ia.uz.zgora.pl,maciejka@astri.uni.torun.pl
}

\begin{abstract}
The aim of this paper is to present two examples of non academic Hamiltonian systems for which the Morales-Ramis theory can be applied effectively. First, we investigate the Gross-Neveu system with $n$ degrees of freedom. Till now it has been proved that this system is not integrable for $n=3$. We give a simple proof that it is not completely integrable for an arbitrary $n \geq 3$. Our second example is a natural generalisation of the Jacobi problem of a material point moving on an ellipsoid. We formulate sufficient conditions for its non-integrability.
\end{abstract}

1. Introduction. It is a very difficult problem to decide whether a dynamical system

$$
\dot{x}=v(x), \quad x \in M^{k},
$$

where $M^{k}$ is an analytic $k$-dimensional complex manifold, possesses or does not possess first integrals. It is well known that having a large enough number of first integrals we can integrate system (1) explicitly. On the other hand, the lack of first integrals can imply a complicated behaviour of phase curves of the system.

One approach to the above problem relates the lack of first integrals to branching of solutions as functions of complex time. Although this idea takes its origin in works of S. V. Kovalevskaya and A. M. Lyapunov, the first mathematical results were obtained quite recently by Ziglin $[14,15]$. He showed that the existence of meromorphic first integrals of system (1) imposes certain conditions on the monodromy group of the variational equation corresponding to a particular solution. Ziglin's basic theorems concern Hamiltonian systems, however, the main part of his theory is applicable for general systems.

In works of J. J. Morales, J.-P. Ramis and M. Singer, Ziglin's theory was extended by a differential Galois approach. For a detailed exposition see [9]. In this paper I present two

2000 Mathematics Subject Classification: Primary 37J30; Secondary 70F20, 34Mxx.

The paper is in final form and no version of it will be published elsewhere. 
applications of this approach for non-academic Hamiltonian systems. Our first example is the Gross-Neveu system with $n$ degrees of freedom. Till now it has been proved that this system is not integrable for $n=3$. This result was obtained in [4] by application of the Ziglin theory. Our second example is a natural generalisation of the Jacobi problem of a material point moving on an ellipsoid. It provides an example of application of Morales-Ramis theory when the problem depends on parameters.

2. Basic facts. The aim of this section is to formulate basic facts concerning the Ziglin and Morales-Ramis theories. This exposition is not formal. All details can be found in [9].

We assume that for a holomorphic system of the form (1) we know a non-equilibrium particular solution $\varphi(t)$. The phase curve $\Gamma$ corresponding to this solution is a Riemann surface. The variational equation corresponding to $\Gamma$ has the form

$$
\dot{\xi}=T(v) \xi, \quad \xi \in T_{\Gamma} M^{k} .
$$

We can reduce the order of this system by one. Let $F:=T_{\Gamma} M^{k} / T \Gamma$ be the normal bundle of $\Gamma$, and $\pi: T_{\Gamma} M^{k} \rightarrow F$ be the projection. Then system (2) induces the following system on $F$

$$
\dot{\eta}=\pi_{\star}\left(T(v) \pi^{-1} \eta\right), \quad \eta \in F,
$$

which is called the normal variational equation along $\Gamma$. In [14] it was shown that if system (1) has $m$ meromorphic functionally independent first integrals, then the monodromy group $\mathcal{M} \subset \mathrm{GL}(k-1, \mathbb{C})$ of $(3)$ has $m$ rational invariants. This statement is also true if instead of the monodromy group we take the differential Galois group of (3), see [9, Chapter IV]. Although this fact gives an excellent tool for proving the non-existence of first integrals for an arbitrary, not necessarily Hamiltonian, system the author knows only one example of its application [16].

It can happen that system (1) possesses $l<k-1$ independent first integrals $F=$ $\left(F_{1}, \ldots, F_{l}\right)$. Then, first we restrict it to the level $F=F(\varphi(t))$, and next we perform the described reduction of the variational equation. We obtain the normal variational equation of order $k-l-1$. This situation happens, in particular, when (1) is Hamiltonian. In this case $k=2 n$, and the monodromy group, as well as the differential Galois group of the normal variational equation are subgroups of $\operatorname{Sp}(2(n-1), \mathbb{C})$.

The basic theorem of Morales-Ramis theory states that if a Hamiltonian system possesses $n$ meromorphic, functionally independent and commuting first integrals in a neighbourhood of $\Gamma$, then the identity component $G^{0}$ of the differential Galois group of the normal variational equation is Abelian. For a detailed exposition and proof see [9].

In order to apply the Morales-Ramis non-integrability theorem it is necessary to know how to check effectively if the identity component of the differential Galois group of a given linear system is Abelian. In general, this is a hard problem. There exist, however, effective methods to investigate this question for lower dimensional systems with rational coefficients. In particular, for second order equations over $\mathbb{C}(x)$ the algorithm of Kovacic [8] allows to decide whether the identity component of the differential Galois group is solvable. For different improvements of the Kovacic algorithm and results concerning 
higher dimensional systems see $[3,10,11,13,12,2]$. Thus, in applications, it is important to find a transformation which does not change the identity component of the differential Galois group and transforms the normal variational equation to an equation with rational coefficients.

To apply the Morales-Ramis theory we have to find a non-constant particular solution of the considered Hamiltonian system. This is a non-trivial task. For all problems investigated till now a particular solution has been found by restricting the Hamiltonian system to a two dimensional invariant symplectic manifold. Thus, in fact, we can always work with a one parameter family of particular solutions. It seems to be typical for cases when Morales-Ramis (or Ziglin) theory is applicable. Thus, it is reasonable to consider to what extend this fact can be used to formulate a stronger version of the non-integrability theorem. In [17] Ziglin showed that several classical problems of mechanics do not possess additional real meromorphic first integrals. In his proof he used explicitly the fact that for those systems we know families of particular solutions containing a homoclinic or heteroclinic orbit.

Another important step is the 'rationalisation' of the normal variational equation. Generally, it is not obvious how to find a transformation of a given linear system to a system with rational coefficients. It seems that it is necessary to develop Kovaciclike algorithms for linear systems with coefficients which are elliptic functions. In some cases, e.g. when the normal variational equation coincides with the Lamé equation, a transformation to a rational form is unnecessary.

A typical system contains parameters and this introduces additional problems when one applies a Kovacic-like algorithm. Typically, we are able to show the non-integrability of the system for almost all parameters' values. For the remaining (usually countably many), the algorithm must be applied separately. This point is very important because, as it is believed, almost all systems are not integrable and thus, among the remaining values of parameters, it is possible to find the ones corresponding to integrable systems. In other words, although a proof of the non-integrability can be a nontrivial task, finding a non-trivially integrable system is the true aim.

3. Gross-Neveu Hamiltonian system. In this section we investigate the so called Gross-Neveu Hamiltonian system given by the Hamiltonian

$$
H=\frac{1}{2} \sum_{i=1}^{n} p_{i}^{2}+\sum_{1 \leq k<j \leq n}\left(u_{j} u_{k}+u_{j}^{-1} u_{k}^{-1}\right)+\sum_{k \neq j} u_{j} u_{k}^{-1},
$$

where we denote $u_{i}:=\exp \left[q_{i}\right]$, for $i=1, \ldots, n$, and $\left(q_{1}, \ldots, q_{n}, p_{1}, \ldots, p_{n}\right)$ are the canonical coordinates on $\mathbb{C}^{2 n}$. This system appears in theoretical physics and its construction is related to the Lie algebra $\operatorname{so}(2 n)$, for details see [1]. We introduce the non-canonical variables $\left(u_{1}, \ldots, u_{n}, v_{1}, \ldots, v_{n}\right)$ where $v_{i}=p_{i} \exp \left[-q_{i}\right], i=1, \ldots, n$. Then the equations of motion have the form

$$
\dot{u}_{j}=v_{j} u_{j}^{2}, \quad \dot{v}_{j}=-v_{j}^{2} u_{j}-\left(1-u_{j}^{-2}\right) \sum_{k \neq j}\left(u_{k}+u_{k}^{-1}\right), \quad j=1, \ldots, n .
$$


We can easily find a particular solution if we notice that the two dimensional plane defined by $v_{l}=0 u_{l}=1$ for $l=2, \ldots, n$ is invariant with respect to the flow generated by (5). On this plane equations (5) read

$$
\dot{u}_{1}=v_{1} u_{1}^{2}, \quad \dot{v}_{1}=-v_{1}^{2} u_{1}-2(n-1)\left(1-u_{j}^{-2}\right) .
$$

As the above system has the first integral

$$
h=\frac{1}{2} v_{1}^{2} u_{1}^{2}+2(n-1)\left(u_{1}+u_{1}^{-1}\right),
$$

we have at our disposal a one parameter family of particular solutions parameterized by the value of $h$. Let $u=u_{1}(t), v=v_{1}(t)$ be a particular solution corresponding to $h=c$. For further considerations we choose $c=4(n-1)$. One can easily notice that the normal variational equation is the direct sum of $(n-1)$ copies of the system

$$
\dot{\xi}=\eta, \quad \dot{\eta}=-2\left[u+u^{-1}+2(n-2)\right] \xi .
$$

It is clear that to prove the non-integrability of the Gross-Neveu system it is enough to show that the identity component of the differential Galois group of (6) is not Abelian. To this end we rewrite (6) in the form

$$
\ddot{\xi}+b \xi=0, \quad b=2\left[u+u^{-1}+2(n-2)\right],
$$

and then we make the transformation

$$
t \rightarrow z:=u(t)
$$

which does not change the identity component of the differential Galois group. We obtain

$$
\xi^{\prime \prime}+p \xi^{\prime}+q \xi=0, \quad ' \equiv \frac{d}{d z},
$$

where

$$
p=\frac{1}{2 z}+\frac{1}{z-1}, \quad q=-\frac{z^{2}+2(m-1) z+1}{2 m z^{2}(z-1)^{2}},
$$

with $m=n-1$. This equation is a Fuchsian one. It has three regular singular points at $z=0, z=1$ and $z=\infty$. Thus it is the Riemann equation and to determine its differential Galois group we can apply the Kimura theorem from [7]. However, in this particular case it is simpler to proceed in a different way. First, we transform the above equation into the standard form

$$
w^{\prime \prime}=r w,
$$

where

$$
w:=\xi \exp \left[\frac{1}{2} \int p\right], \quad r=\frac{1}{2} p^{\prime}+\frac{1}{4} p^{2}-q .
$$

Simple calculations show that

$$
r=\frac{8-3 m}{16 m z^{2}}+\frac{3}{4 z}+\frac{3}{4(z-1)^{2}}-\frac{3}{4(z-1)},
$$

and exponents $\rho_{i}^{ \pm}$at singular points $i \in\{0,1, \infty\}$ are

$$
\rho_{1}^{ \pm}=-\frac{1}{2}, \frac{3}{2}, \quad \rho_{0}^{ \pm}=-\rho_{\infty}^{ \pm}=\frac{1}{2}(1 \pm \Delta), \quad \Delta=\sqrt{\frac{1}{4}+\frac{2}{m}} .
$$

We have the following. 
Lemma 1. The differential Galois group $G$ of (7) for $m>1$ is not conjugate to a finite imprimitive subgroup of $\mathrm{SL}(2, \mathbb{C})$.

Proof. It is easy to show that $\Delta \notin \mathbb{Q}$ for $m>1, m \in \mathbb{N}$, and thus $\rho_{0}^{ \pm} \notin \mathbb{Q}$ and $\rho_{\infty}^{ \pm} \notin \mathbb{Q}$. Hence, by Theorem 3.6 in [11], the necessary conditions for $G$ to be a finite imprimitive subgroup of $\operatorname{SL}(2, \mathbb{C})$ are not satisfied, i.e. case 3 of the Kovacic algorithm is excluded, see Theorem on page 8 in [8].

Lemma 2. The differential Galois group $G$ of (7) for $m>1$ is conjugate to a triangular subgroup of $\mathrm{SL}(2, \mathbb{C})$ or it is $\mathrm{SL}(2, \mathbb{C})$.

Proof. Let us investigate the local monodromy around $z=1$. At this point the difference between exponents $s=\rho_{1}^{+}-\rho_{1}^{-}=2$. One local solution has the form

$$
w_{1}(z)=(z-1)^{\rho_{1}^{+}} f(z), \quad f(z)=1+\sum_{k=}^{\infty} f_{k}(z-1)^{k},
$$

where the series defining $f(z)$ converges in a neighbourhood of $z=1$. The second solution, linearly independent of $w_{1}(z)$ is defined by the integral

$$
w_{2}(z)=w_{1}(z) \int^{z} \frac{\mathrm{d} \zeta}{w_{1}(\zeta)^{2}}=w_{1}(z) \int^{z}(\zeta-1)^{-s-1} \frac{\mathrm{d} \zeta}{f(\zeta)^{2}} .
$$

Let us denote

$$
\frac{1}{f(z)^{2}}=1+\sum_{k=1}^{\infty} g_{k}(z-1)^{k}
$$

Then the solution $w_{2}(z)$ can be written in the form

$$
w_{2}(z)=w_{1}(z) g_{s} \ln (z-1)+(z-1)^{\rho_{1}^{-}} v(z),
$$

where $v(z)$ is holomorphic in a neighbourhood of $z=1$. The form of the local monodromy depends on the presence of the logarithmic term. To check if it is present in our case we have to calculate if $g_{2} \neq 0$. It is easy to calculate that

$$
f_{1}=-\frac{1}{4}, \quad f_{2}=\frac{2+3 m}{32 m}, \quad g_{2}=3 f_{1}^{2}-2 f_{2}=-\frac{1}{8 m} .
$$

Thus, the monodromy $M_{1}$ corresponding to a small loop around $z=1$ has the form

$$
M_{1}=\left[\begin{array}{cc}
1 & 2 \pi \mathrm{i} \\
0 & 1
\end{array}\right]
$$

The monodromy group $M$ is a subgroup of $G$ (in our case it generates topologically $G$ ). A subgroup of $\mathrm{SL}(2, \mathbb{C})$ generated by a triangular matrix cannot be finite and thus the differential Galois group cannot be finite as it contains the triangular matrix $M_{1}$. This is in fact another proof of Lemma 1 . There is only one possibility that we have to exclude. The group $G$ can be conjugate to a subgroup of

$$
D^{\dagger}=\left\{\left[\begin{array}{cc}
c & 0 \\
0 & c^{-1}
\end{array}\right] \mid c \in \mathbb{C}^{*}\right\} \cup\left\{\left[\begin{array}{cc}
0 & c \\
c^{-1} & 0
\end{array}\right] \mid c \in \mathbb{C}^{*}\right\},
$$

see $\left[8\right.$, p. 7]. However, the group $D^{\dagger}$ cannot contain a non-diagonalizable triangular matrix.

Basing on the above lemmas we are able to show that: 
Theorem 1. For $n>2$ the Gross-Neveu system is not integrable.

Proof. Assume that the Gross-Neveu system is integrable. Then the identity component $G^{0}$ of $(7)$ is Abelian. From Lemma 1 and Lemma 2 it follows that $G$ is conjugate to a subgroup of the triangular group. $G$ cannot be a diagonal as it contains $M_{1}$. Thus, it must be a proper subgroup of the triangular group. However, in this case the elements of $G$ have eigenvalues which are $k$-th roots of unity for certain $k \in \mathbb{N}$, see Lemma 4.2 in [10]. However, the local monodromy matrix $M_{0} \in G$ corresponding to the singular point $z=0$ has eigenvalues $\lambda^{ \pm}=\exp \left[2 \pi \mathrm{i} \rho_{0}^{ \pm}\right]$. Hence $\rho_{0}^{ \pm} \notin \mathbb{Q}$, and thus $\lambda^{ \pm}$are not roots of unity and $G^{0}$ is not Abelian. This contradiction finishes the proof.

In [4] it was proved that the Gross-Neveu system is not integrable for $n=3$ with the help of an important extension of the Ziglin theorem to a case when the monodromy group contains only resonant elements of special form. However, this extension works only for a system with three degrees of freedom. The reader will find it interesting to compare the reasoning in [4] with our, very simple, considerations.

4. Generalised Jacobi problem. Let a point $P$ of unit mass move on an ellipsoid

$$
\mathcal{E}:=\left\{\boldsymbol{x} \in \mathbb{R}^{3} \mid\langle\boldsymbol{x}, \boldsymbol{A} x\rangle-1=0\right\}
$$

under the action of a force given by the potential

$$
V(\boldsymbol{x}):=\frac{1}{2}\langle\boldsymbol{x}, \boldsymbol{B} x\rangle,
$$

where $\boldsymbol{A}=\operatorname{diag}\left(a_{1}, a_{2}, a_{3}\right), a_{1} \geq a_{2} \geq a_{3}>0, \boldsymbol{B}$ is a symmetric matrix, and $\langle\cdot, \cdot\rangle$ denotes the scalar product in $\mathbb{R}^{3}$.

Equations of motion of the point can be written in the form

$$
\dot{\boldsymbol{x}}=\boldsymbol{y}, \quad \dot{\boldsymbol{y}}=-\boldsymbol{B} \boldsymbol{x}-\lambda \boldsymbol{A} \boldsymbol{x},
$$

where the Lagrange multiplier is

$$
\lambda:=\frac{\langle\boldsymbol{y}, \boldsymbol{A} y\rangle-\langle\boldsymbol{B} x, \boldsymbol{A} x\rangle}{\langle\boldsymbol{A} x, \boldsymbol{A} x\rangle} .
$$

We consider equations (10) on the tangent bundle

$$
T \mathcal{E}=\left\{(\boldsymbol{x}, \boldsymbol{y}) \in \mathbb{R}^{6} \mid \boldsymbol{x} \in \mathcal{E}, \quad\langle\boldsymbol{y}, \boldsymbol{A} x\rangle=0\right\} .
$$

On $T \mathcal{E}$ equations (10) possess the energy integral

$$
E(\boldsymbol{x}, \boldsymbol{y})=\frac{1}{2}\langle\boldsymbol{y}, \boldsymbol{y}\rangle+V(\boldsymbol{x}) .
$$

As the system has two degrees of freedom, for its integrability it is necessary to have one additional first integral. All known integrable cases are the following:

1. Jacobi case: $\boldsymbol{B}=b \boldsymbol{E}$ where $b \in \mathbb{R}$ and $\boldsymbol{E}$ is the unit matrix,

2. Neumann case: $a_{1}=a_{2}=a_{3}$,

3. symmetric case: $\boldsymbol{B}=\operatorname{diag}\left(b_{1}, b_{2}, b_{3}\right), a_{i}=a_{j}, b_{i}=b_{j}, i \neq j$,

4. 'no tangent force' case: $\boldsymbol{B}=\alpha \boldsymbol{A}, \alpha \in \mathbb{R}$. 
In what follows, we consider the case of diagonal potential $\boldsymbol{B}=\operatorname{diag}\left(b_{1}, b_{2}, b_{3}\right)$. Then, all the above integrable cases satisfy the condition

$$
b_{1}\left(a_{2}-a_{3}\right)+b_{2}\left(a_{3}-a_{1}\right)+b_{3}\left(a_{1}-a_{2}\right)=0 .
$$

When this condition is satisfied then the Hamilton-Jacobi equation for the Jacobi and the 'no tangent force' cases separates in the ellipsoidal coordinates. This fact was discovered by C. G. J. Jacobi who introduced the ellipsoidal coordinates especially for the purpose of explicit integration of the geodesic motion on an ellipsoid, see [6].

From now on we will work with the complexified system (10), i.e. we assume that $(\boldsymbol{x}, \boldsymbol{y}) \in \mathbb{C}^{6}$ and $t \in \mathbb{C}$.

It is obvious that the three hyper-planes

$$
\Pi_{k}=\left\{(\boldsymbol{x}, \boldsymbol{y}) \in \mathbb{C}^{6} \mid x_{k}=0, \quad y_{k}=0\right\}, \quad k=1,2,3,
$$

are invariant with respect to the flow generated by (10). System (10) restricted to $\Xi_{k}:=$ $\Pi_{k} \cap T \mathcal{E}$ describes the motion of a point moving in an ellipse. As it is a system with one degree of freedom, we can integrate it explicitly. In this way we are able to obtain three families of particular solutions of (10) which are used in our application of Morales-Ramis theory.

Let us consider a constant energy level

$$
\Sigma_{h}:=\{(\boldsymbol{x}, \boldsymbol{y}) \in T \mathcal{E} \mid E(\boldsymbol{x}, \boldsymbol{y})=h\} .
$$

We assume that $h \in \mathbb{C}$ is such that for $k=1,2,3$, the intersection $\Sigma_{h} \cap \Xi_{k}$ is non-empty and contains a solution

$$
\boldsymbol{x}=\boldsymbol{\varphi}(t), \quad \boldsymbol{y}=\dot{\varphi}(t), \quad t \in \mathbb{C},
$$

which is not an equilibrium. We denote by $\Gamma_{k}^{h}$ the Riemann surface associated with the selected solution lying in $\Xi_{k}$. The linear variational equation along solution (17) can be written in the form

$$
\ddot{\boldsymbol{\xi}}+[\boldsymbol{B}+\lambda \boldsymbol{A}] \boldsymbol{\xi}+\left[\left\langle\nabla_{\boldsymbol{x}} \lambda, \boldsymbol{\xi}\right\rangle+\left\langle\nabla_{\boldsymbol{y}} \lambda, \dot{\boldsymbol{\xi}}\right\rangle\right] \boldsymbol{A} \boldsymbol{\varphi}(t)=\mathbf{0},
$$

where $\lambda, \nabla_{\boldsymbol{x}} \lambda$ and $\nabla_{\boldsymbol{y}} \lambda$ are calculated at $(\boldsymbol{x}, \boldsymbol{y})=(\boldsymbol{\varphi}(t), \dot{\boldsymbol{\varphi}}(t))$, and $\boldsymbol{\xi}=\left(\xi_{1}, \xi_{2}, \xi_{3}\right)$. The considered solution lies on $\Xi_{k}$ and for it we have $\varphi_{k}(t) \equiv 0$ and $\dot{\varphi}_{k}(t) \equiv 0$. Thus, the normal variational equation for this solution corresponds to the $k$-th component of $\boldsymbol{\xi}$ and it has the form

$$
\ddot{\xi}_{k}+\left[b_{k}+\lambda a_{k}\right] \xi_{k}=0 .
$$

This equation is considered as an equation on $\Gamma_{k}^{h}$, where $t$ is taken as a local coordinate. We transform it choosing another local coordinate $z$ in such a way that the resulting equation will have coefficients in $\mathbb{C}(z)$. To simplify further exposition we restrict our attention to the case $k=3$. Notice that $\Gamma_{3}^{h}$ is an algebraic curve given by the following equations:

$$
\begin{aligned}
& a_{1} x_{1}^{2}+a_{2} x_{2}^{2}=1, \quad x_{3}=0, \quad y_{3}=0, \\
& a_{1} x_{1} y_{1}+a_{2} x_{2} y_{2}=0, \quad y_{1}^{2}+y_{2}^{2}+b_{1} x_{1}^{2}+b_{2} x_{2}^{2}=2 h
\end{aligned}
$$

As a local coordinate on it we can take e.g. $x_{1}^{2}$. Thus we make a transformation

$$
t \rightarrow z=\varphi_{1}(t)^{2} \text {. }
$$


First let us note that after this transformation $\lambda=\lambda(\varphi(t), \dot{\varphi}(t))$ is a rational function of $z$ and we denote it $\lambda(z)$. Moreover, we have

$$
\begin{aligned}
\frac{\mathrm{d}}{\mathrm{d} t} & =2 \varphi_{1}(t) \dot{\varphi}_{1}(t) \frac{\mathrm{d}}{\mathrm{d} z}, \\
\frac{\mathrm{d}^{2}}{\mathrm{~d} t^{2}} & =\left[2 \dot{\varphi}_{1}(t)^{2}-2\left(\lambda(z) a_{1}+b_{1}\right) z\right] \frac{\mathrm{d}}{\mathrm{d} z}+4 z \dot{\varphi}_{1}(t)^{2} \frac{\mathrm{d}^{2}}{\mathrm{~d} z^{2}} .
\end{aligned}
$$

To obtain the last relation we use the explicit form of the right hand side of equation (10) for $\ddot{x}_{1}$. From (20) it follows also that $\dot{\varphi}_{1}(t)^{2}=y_{1}^{2}$ is a rational function of $z$ which we denote $y_{1}(z)^{2}$. The normal variational equation (19) after transformation (21) reads

$$
\frac{\mathrm{d}^{2} \xi_{3}}{\mathrm{~d} z^{2}}+p(z) \frac{\mathrm{d} \xi_{3}}{\mathrm{~d} z}+q(z) \xi_{3}=0
$$

where

$$
p(z)=\frac{1}{2}\left(\frac{1}{z}-\frac{\lambda(z) a_{1}+b_{1}}{y_{1}(z)^{2}}\right), \quad q(z)=\frac{\lambda(z) a_{3}+b_{3}}{4 z y_{1}(z)^{2}} .
$$

Making the change of the dependent variable

$$
w=\xi_{3} \exp \left[-\frac{1}{2} \int_{z_{0}}^{z} p(\zeta) \mathrm{d} \zeta\right]
$$

we transform (23) into the standard form

$$
\frac{\mathrm{d}^{2} w}{\mathrm{~d} z^{2}}-J(z) w=0
$$

where

$$
J(z)=-q(z)+\frac{1}{4} p(z)^{2}+\frac{1}{2} \frac{\mathrm{d} p(z)}{\mathrm{d} z} .
$$

Finally, in order to reduce the number of parameters we perform the re-scaling $z \rightarrow z / a_{1}$ and we obtain

$$
\frac{\mathrm{d}^{2} w}{\mathrm{~d} z^{2}}-r(z) w=0
$$

where

$$
r(z)=\frac{1}{a_{1}^{2}} J\left(z / a_{1}\right) .
$$

Now the rational function $r(z)$ depends on five parameters, namely

$$
\alpha_{k}=\frac{a_{k}}{a_{1}}, \quad \beta_{k}=\frac{b_{k}}{b_{1}}, \quad k=2,3, \quad \text { and } \quad \mu=2 \frac{h a_{1}}{b_{1}} .
$$

In what follows we assume that:

1. the ellipsoid is tri-axial, i.e. $\alpha_{k} \neq 1$ for $k=2,3$, and $\mu \neq 1$,

2. we exclude the 'no tangent force' case,

3. $\beta_{3} \alpha_{2}-\beta_{2} \alpha_{3} \neq 0$.

The case of an axially symmetric ellipsoid, as well as that excluded by condition 3 above must be considered separately.

It is easy to notice that equation (27) is Fuchsian. It has five singular points

$$
z_{1}=0, \quad z_{2}=1, \quad z_{3}=\frac{\beta_{2}-\mu \alpha_{2}}{\beta_{2}-\alpha_{2}}, \quad z_{4}=\frac{\alpha_{2}}{\alpha_{2}-1}, \quad z_{5}=\infty
$$


with exponents $\rho_{i}^{+}, \rho_{i}^{-}($see $[5])$

$$
\begin{gathered}
\rho_{i}^{ \pm}=\frac{1}{4}, \frac{3}{4}, \quad \text { for } \quad i=1,2,3, \\
\rho_{4}^{ \pm}=-\frac{1}{4}, \frac{5}{4}, \quad \rho_{5}^{ \pm}=-\frac{1}{2}\left(1 \pm \Delta_{3}\right),
\end{gathered}
$$

where

$$
\Delta_{3}=\sqrt{\frac{\alpha_{3}\left(1-\beta_{2}\right)+\beta_{3}\left(\alpha_{2}-1\right)}{\alpha_{2}-\beta_{2}}} .
$$

We can repeat the above calculation for $\Gamma_{1}^{h}$ and $\Gamma_{2}^{h}$. In both cases we obtain a Fuchsian equation of the form (27) with five singular points. In all cases the exponents of singular points are the same except $z_{5}=\infty$. For $\Gamma_{1}^{h}$ and $\Gamma_{2}^{h}$ they are

$$
\rho_{5}^{ \pm}=-\frac{1}{2}\left(1 \pm \Delta_{k}\right), \quad k=1,2
$$

where

$$
\Delta_{1}=\sqrt{\frac{\alpha_{2}-\beta_{2}+\beta_{3}-\alpha_{3}}{\beta_{3} \alpha_{2}-\beta_{2} \alpha_{3}}}, \quad \Delta_{2}=\sqrt{\frac{\alpha_{2}\left(\beta_{3}-1\right)+\beta_{2}\left(1-\alpha_{3}\right)}{\beta_{3}-\alpha_{3}}} .
$$

The main result of this section is the following theorem.

THEOREM 2. If there exists $k \in\{1,2,3\}$ such that

$$
\Delta_{k} \neq \frac{p}{q}, \quad p \in \mathbb{N}, \quad q=1, \ldots, 6,
$$

then system (10) is not integrable.

In the above theorem it is understood that the three assumptions given above are satisfied.

Proof. Assume that system (10) is integrable. Then for every particular solution the identity component $G^{0}$ of the differential Galois group of the respective NVE is Abelian. We show that if condition (29) is satisfied, then, for NVEs corresponding to $\Gamma_{k}^{h}, k=1,2,3$, we have $G=G^{0}=\mathrm{SL}(2, \mathbb{C})$, except for one special value of $\mu$. Our proof is heavily based on the Kovacic algorithm [8] and some computer algebra calculations.

For all three cases NVE is Fuchsian so all four possibilities given by Lemma on p. 7 in [8] can occur. Thus, we have to follow the Kovacic algorithm from Case I to Case III. We are going to consider NVE corresponding to $\Gamma_{3}^{h}$, but for the other two the procedure is the same.

Case $I$. We check if $G$ can be conjugate to a triangular subgroup of $\operatorname{SL}(2, \mathbb{C})$, or, equivalently, if (27) has a solution of the form $w=P \exp \int \omega$, where $P \in \mathbb{C}[z]$ and $\omega \in \mathbb{C}(z)$. According to the algorithm described on pp. 11-12 in [8], for each singular point we calculate the auxiliary set $E_{i}$. In our case we have

$$
\begin{aligned}
& E_{5}=\left\{\frac{1}{2}\left(1 \pm \Delta_{3}\right)\right\}, \quad E_{4}=\left\{-\frac{1}{4}, \frac{5}{4}\right\}, \\
& E_{i}=\left\{\frac{1}{4}, \frac{3}{4}\right\}, \quad \text { for } \quad i=1,2,3 .
\end{aligned}
$$


Let $E=\prod E_{i}$ be the Cartesian product of all $E_{i}$. Then

$$
\operatorname{deg} P:=d(e):=e_{5}-\sum_{i=1}^{4} e_{i},
$$

for some $e=\left(e_{1}, \ldots, e_{5}\right) \in E$. However, for an arbitrary $e \in E$ we have

$$
d(e)=-\frac{l}{k} \pm \frac{1}{2} \Delta_{3}, \quad k=1,2,4, \quad l \in \mathbb{N}_{0}:=\mathbb{N} \cup\{0\} .
$$

Thus, if $\Delta_{3}$ satisfies (29), then $d(e) \notin \mathbb{N}_{0}$, and Case I cannot occur.

Case II. We check if $G$ can be an imprimitive subgroup of $\operatorname{SL}(2, \mathbb{C})$, or, equivalently, if there exists a solution of the form $w=\exp \int \omega$ where $\omega$ belongs to the quadratic extension of $\mathbb{C}(x)$. Now, the sets $E_{i}$ are the following (see p. 18 in [8]):

$$
E_{1}=E_{2}=E_{3}=\{1,2,3\}, \quad E_{4}=\{-1,2,5\}
$$

and

$$
E_{5}=\left\{2,2 \pm 2 \Delta_{3}\right\} \cap \mathbb{Z}=\{2\}
$$

because $\Delta_{3}$ satisfies (29). Now, we look for $e \in E$ such that $d_{2}(e):=d(2) / 2 \in \mathbb{N}_{0}$. For $e=(1,1,1-1,2), d_{2}(e)=0$, and this is the only possibility when $d_{2}(e) \in \mathbb{N}_{0}$. Now, we have to check if there exists a monic polynomial $P$ of degree 0 satisfying a certain linear differential equation, (see p. 18 in [8]). In this case it can be checked with the help of a computer algebra system that such a solution exists only if

$$
\mu=\frac{\beta_{2}-\alpha_{2}^{2}}{\alpha_{2}\left(1-\alpha_{2}\right)} .
$$

If we exclude this value of $\mu$ then Case II cannot occur.

Case III. We check if $G$ can be a finite subgroup of $\operatorname{SL}(2, \mathbb{C})$, or, equivalently, if there exists a solution of the form $w=\exp \int \omega$ where $\omega$ belongs to an algebraic extension of $\mathbb{C}(z)$ of degree $n=4,6$ or 12 . Each value of $n$ must be considered separately, see pp. $22-23$ in [8].

Subcase $n=4$. Now the sets $E_{i}$ are the following:

$$
E_{1}=E_{2}=E_{3}=\{3,6,9\}, \quad E_{4}=\{-3,6,15\}, \quad E_{5}=\{6\} .
$$

To calculate $E_{i}$ we made use of condition (29). It is easy to notice that there is no $e \in E$ such that $d_{4}(e):=d(e) / 3 \in \mathbb{N}_{0}$. Thus this subcase cannot occur.

Subcase $n=6$. We have now

$$
E_{1}=E_{2}=E_{3}=\{3,4,5,6,7,8,9\} \quad E_{4}=\{-3,0,3,6,9,12,15\}, \quad E_{5}=\{6\},
$$

and there is only one $e \in E$ such that $d_{6}(e):=d(e) / 2 \in \mathbb{N}_{0}$. Namely, for $e=(3,3,3,-3,6)$, we have $d_{6}(e)=0$. As in Case II we have to check if the polynomial $P=1$ satisfies a certain differential equation, if it does not, then this subcase is excluded. Using computer algebra we checked that $P=1$ satisfies this equation only for the value of $\mu$ given by (31). As we have already excluded this value of $\mu$, the subcase $n=6$ cannot occur.

Subcase $n=12 . E_{i}$ are the same as in the previous subcase. Again there is only one $e \in E$, exactly the same as in the previous subcase, such that $d_{12}(e):=d(e) \in \mathbb{N}_{0}$. With the help of a computer algebra program we checked that an appropriate equation has 
no polynomial solution of degree 0 except for the value of $\mu$ given by (31). Thus, this subcase, as well as the whole Case III must be excluded.

From the above considerations it follows that if $\mu$ is not given by (31), then the considered NVE has no Liouvillian solution and its differential Galois group is $\operatorname{SL}(2, \mathbb{C})$. Let us notice that we can always choose the curve $\Gamma_{3}^{h}$ such that $\mu$, which is a re-scaled value of $h$, does not satisfy (31). Thus, for this choice the identity component of the differential Galois group of NVE is not Abelian. This is a contradiction with our assumption that the system is integrable.

If, instead of $\Gamma_{3}^{h}$ in the above proof, we choose $\Gamma_{1}^{h}$ or $\Gamma_{2}^{h}$, different values of $\mu$ have to be excluded. It is interesting to see if it can happen that all these three values coincide. If this is possible then for this particular choice for all three solutions $G^{0}$ is Abelian. A simple analysis shows that this can happen if and only if

$$
b_{1} a_{2} a_{3}\left(a_{3}-a_{2}\right)+b_{2} a_{1} q_{3}\left(a_{1}-a_{3}\right)+b_{3} a_{1} a_{2}\left(a_{2}-a_{1}\right)=0 .
$$

Of course, even if this relation is satisfied, we cannot expect that the system is integrable. However, restricting it to the energy level corresponding to the distinguished value of $\mu$, we can obtain an integrable system. In other words, we conjecture that the original system can be integrable on the particular level of the energy integral.

We remark also that the investigations of cases which do not satisfy (29) seem to be considerably more difficult than that presented above.

The result of this section is an effect of my common work with B. S. Bardin.

Acknowledgements. I would like to thank Tomasz Stachowiak who pointed out an error in the first version of this paper.

\section{References}

[1] M. Adler and P. van Moerbeke, Kowalewski's asymptotic method, Kac-Moody Lie algebras and regularization, Comm. Math. Phys. 83 (1982), 83-106.

[2] D. Boucher, Sur les équations différentielles linéaires paramétrées, une application aux systèmes hamiltoniens, PhD thesis, Université de Limoges, France, 2000.

[3] A. Duval and M. Loday-Richaud, Kovačič's algorithm and its application to some families of special functions, Appl. Algebra Engrg. Comm. Comput. 3 (1992), 211-246.

[4] E. Horozov, On the nonintegrability of Gross-Neveu models, Ann. Physics 174 (1987), 430-441.

[5] E. L. Ince, Ordinary Differential Equations, Dover Publications, New York, 1944.

[6] C. G. J. Jacobi, Vorlesungen über Dynamik, G. Reimer, Berlin, 1884.

[7] T. Kimura, On Riemann's equations which are solvable by quadratures, Funkcial. Ekvac. $12(1969 / 70), 269-281$.

[8] J. J. Kovacic, An algorithm for solving second order linear homogeneous differential equations, J. Symbolic Comput. 2 (1986), 3-43.

[9] J. J. Morales Ruiz, Differential Galois Theory and Non-Integrability of Hamiltonian Systems, Birkhäuser Verlag, Basel, 1999. 
[10] M. F. Singer and F. Ulmer, Liouvillian and algebraic solutions of second and third order linear differential equations, J. Symbolic Comput. 16 (1993), 37-73.

[11] M. F. Singer and F. Ulmer, Necessary conditions for Liouvillian solutions of (third order) linear differential equations, Appl. Algebra Engrg. Comm. Comput. 6 (1995), 1-22.

[12] M. van Hoeij, J.-F. Ragot, F. Ulmer and J.-A. Weil, Liouvillian solutions of linear differential equations of order three and higher, J. Symbolic Comput. 28 (1998), 589-609.

[13] F. Ulmer and J.-A. Weil, Note on Kovacic's algorithm, J. Symbolic Comput. 22 (1996), 179-200.

[14] S. L. Ziglin, Branching of solutions and non-existence of first integrals in Hamiltonian mechanics. I, Funktsional. Anal. i Prilozhen. 16 (3) (1982), 30-41 (in Russian).

[15] S. L. Ziglin, Branching of solutions and non-existence of first integrals in Hamiltonian mechanics. II, Funktsional. Anal. i Prilozhen. 17 (1) (1983), 8-23 (in Russian).

[16] S. L. Ziglin, On the nonintegrability of the $A B C$-flow for $A=B$, Funktsional. Anal. i Prilozhen. 30 (2) (1996), 80-81 (in Russian).

[17] S. L. Ziglin, On the absence of a real-analytic first integral in some problems of dynamics, Funktsional. Anal. i Prilozhen. 31 (1) (1997), 3-11 (in Russian). 